\title{
Zotero for Personal Image Management
}

Alexander Watkins, University of Colorado Boulder

\begin{abstract}
Digital software solutions for personal image collections have lagged behind digital asset management tools for institutional collections. Scholars often need a solution that can store and organize images, associate metadata, and output data in useful ways. Zotero, the open-source citation management software, is emerging as useful tool for personal image management and library organization. The author describes the applications and advantages of Zotero for personal image collections and its potential as a method by which personal and institutional collections may be more closely integrated.
\end{abstract}

[This article is an expanded version of a presentation given as part of the Emerging Technology Forum at the ARLIS/NA annual conference held in Pasadena, California, in April 2013.]

\section{N T ROD U CT IO N}

Images are a form of data. They can be the basic unit of analysis for scholars or essential forms of illustration; images form key parts of their books, articles, creative works, presentations, and teaching. Over the course of a career, scholars can amass huge personal image collections that include photographs taken on trips, digitized slides, those perfect teaching images, or purchased images for publication. The expansion of digital images means that librarians and visual resource professionals are needed more than ever to help users obtain, manage, and use large quantities of visual materials. In an ideal world, images and their metadata would flow from institutional and subscription databases to personal collections and into papers and presentations. Well-documented and well-preserved images in personal collections would also be shared and incorporated into an institution's collection.

The missing link is one over which information professionals have the least control: the management of personal collections. This gap can be filled by Zotero, a scholar-built tool for organizing research with extensive capabilities for storing and 


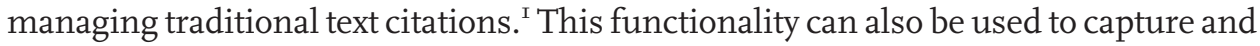
manage images. The software can pull images and metadata from databases and help users keep their collections organized. It empowers users to plug those images into papers or presentations along with formatted citations. For users with unique personal collections, it can provide better backups and documentation, allowing these resources to be preserved and shared. Information professionals can teach Zotero as part of any personal image management program, but they also can become part of the Zotero community to help create a better, more powerful tool for working with images.

\section{PERSONAL IMAGE COLLECTIONS}

Art historians, artists, archaeologists, and architects are often most associated with image use in teaching, scholarship, and design. A study by Joan Beaudoin and Jessica Brady looked at these four groups and found that all make heavy use of personal image collections to supplement institutional and subscription databases, with the heaviest use by artists. ${ }^{2}$ Individual image collections are not limited to these disciplines, as 44 percent of both faculty and students at Pennsylvania State University were discovered to have personal image collections. ${ }^{3}$ Users found that having a personal collection bypassed the shortcomings they encountered in other methods of finding images. ${ }^{4}$ Those scholars with large personal image libraries were shown in the Penn State study to be frequent users of institutionally managed collections as well. ${ }^{5}$ In most cases personal image collections do not compete with institutional resources, but rather they merge as researchers download and store images from a variety of locations. Scholars use a wide range of methods to procure images, including frequenting their visual resources center, scanning books, using databases and search engines, as well as taking photographs in situ. ${ }^{6}$ These personal libraries grow as users bring together on their own computers images from disparate sources, repurposing them for their own projects. ${ }^{7}$

It is no surprise that so many scholars and students maintain personal collections, since images are absolutely vital to their teaching, research, creative processes, and workflow. This need has not slowed with the advent of digital images-in fact, it has sped up. The ease by which digital images may be created, copied, collected, and shared has led to an increase in the number of personal collections. ${ }^{8}$ Image users need to supplement subscription databases like ARTstor which still often lack a suf-

\footnotetext{
I. Readers unfamiliar with using Zotero as a citation manager can get a quick introduction here: "Quick Start Guide [Zotero Documentation]," https://www.zotero.org/support/quick_start_guide.

2. Joan E. Beaudoin and Jessica Evans Brady, "Finding Visual Information: A Study of Image Resources Used by Archaeologists, Architects, Art Historians, and Artists," Art Documentation 30, no. 2 (October I5, 20II): 3I. 3. John Attig, Ann Copeland, and Michael Pelikan, "Context and Meaning: The Challenges of Metadata for a Digital Image Library within the University," College a Research Libraries 65, no. 3 (May 2004): 253.

4. Beaudoin and Brady, "Finding Visual Information," 30.

5. Henry A. Pisciotta et al., "Penn State's Visual Image User Study," portal: Libraries and the Academy 5, no. I (2005): 35, doi: I0.I353/pla.2005.00II.

6. Beaudoin and Brady, "Finding Visual Information," 25.

7. "Advocating for Visual Resources Management in Educational and Cultural Institutions," Visual Resources Association Bulletin 36, no. 3 (Winter 2009): 60.

8. Ibid., 74 .
} 
ficient number of images of works by important artists, specialized media, and contemporary art. ${ }^{9}$ Scholars often require unique images for teaching and high-quality images for publishing and research. Artists and architects often gather inspiring or interesting example images from which to work. ${ }^{\text {Io }}$ New areas of knowledge and inquiry, such as computer modeling and data visualization, create fresh needs. Teachers, students, and museum curators often devise new ways in which images are used. ${ }^{\text {II }}$

Personal collections are no substitute for institutional support, but instead there is a symbiotic relationship. Users gather images from larger collections, and in turn they contribute their original work to visual resource centers, including original photography of high quality and, in some cases, authoritative descriptive data. ${ }^{\mathrm{I2}}$ However, this flow of images is often impeded because downloaded images become detached from the metadata, and personal collections are not adequately documented for uploading. A lack of organization and rigor in personal image collections prevents this ideal reciprocity.

Anecdotes and personal experiences abound of personal image collections placed in random folders that use whatever file name the scanner assigned. More commonly than not, the files have minimal descriptive and source information and are stored on less-than-ideal devices with inadequate backups. ${ }^{\mathrm{I}}$ Locating images on a computer without an organizational structure in a large collection can be difficult and timeconsuming. ${ }^{\text {I4 }}$ Memory suffices for images with which scholars are currently working, but over time it becomes difficult to remember the actual location of a file let alone the contents of a larger collection. ${ }^{15}$ Because of the importance of personal image collections and the failure of a standard file system to manage images, programs and services that facilitate the organization of image collections can be an important tool for image users' workflows and personal archives.

The continually expanding use of digital images in academia offers an opportunity for librarians and visual resource professionals to share their expertise in organization, metadata, and collection-management tools with users. Teaching personal image management is already happening in many libraries and visual resources centers, but many of the tools for organizing personal image collections are woefully inadequate.

\section{PERSONAL IMAGE MANAGERS}

There are several digital tools that bring order to personal image collections. Virginia Hall, senior instructional designer at Johns Hopkins University, maintains a site with

9. Allan Kohl, "The Pedagogical and Research Value of Images and Locally Managed Image Collections: Library, Visual

Resources, and Faculty Perspectives," Visual Resources Association Bulletin 37, no. 3 (Winter 2010):52.

Io. Beaudoin and Brady, "Finding Visual Information," 25.

II. "Advocating for Visual Resources Management in Educational and Cultural Institutions," 6o.

I2. Ibid., GI.

I3. Kohl, "The Pedagogical and Research Value of Images and Locally Managed Image Collections," 55; "Advocating for Visual Resources Management in Educational and Cultural Institutions," 6I.

I4. Chun-Nan Lin, Chih-Fong Tsai, and Jinsheng Roan, "Personal Photo Browsing and Retrieval by Clustering Techniques: Effectiveness and Efficiency Evaluation," Online Information Review 32, no. 6 (2008): 759 .

I5. Hung-Hsuan Huang, Yasuyuki Sumi, and Toyoaki Nishida, "Personal Image Repositories as Externalized Memory Spaces," International Journal of Knowledge Based Intelligent Engineering Systems Io, no. 2 (June 2006): I70. 


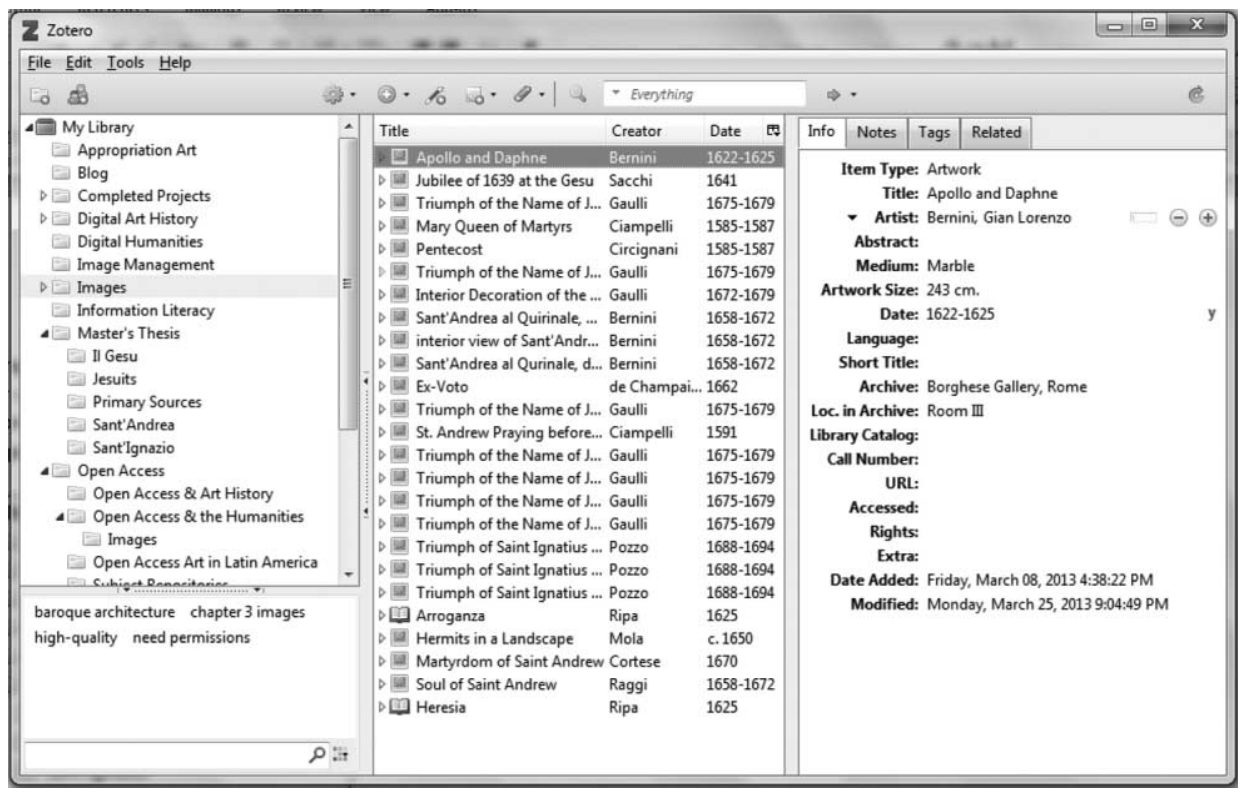

Figure 1. Zotero's interface being used for image management. Please see the online edition of Art Documentation for a color version of this image.

resources and instructions for using many different programs. ${ }^{16}$ Some of the more lightweight solutions include Flickr, Picasa, and iPhoto. Both Picasa and iPhoto overlay the computer's file structure as an organization layer, but they lack robust metadata. Flickr has more metadata options; however, most are designed to document the photography and rights. Title, description, and tags are the only fields it provides for descriptive metadata.

More powerful tools include Adobe Lightroom and digital asset management systems such as CONTENTdm, Omeka, and CollectiveAccess. Lightroom, while powerful, is expensive and designed for professional photographers. Omeka and CollectiveAccess are wonderful open-source tools, but since they are aimed at institution-level collections they have more significant technological barriers and may be overkill for an individual. An Omeka.com account can be used to create a personal digital library, but at a cost. It is better suited for people aiming to create a web-accessible database. Another option is Endnote, which can support images and figures, but it is not free and is limited both in its ability to take in image data as well as in its image-citation capability. Endnote has no plans to expand this capability. Since Zotero is stable open-source software, it can be transformed by interested users into a continually improving resource for image collection and citation (Figure I).

Zotero was built by and for scholars and therefore has much more power and extensibility then the lightweight programs aimed at the casual photographer. It is also designed to be used by a single person with relative ease, and therefore presents a lower entry barrier than server-based programs like Omeka or CollectiveAccess.

I6. “Virginia (Macie) Hall,” https://jshare.johnshopkins.edu/vhall2/public_html/. 
Despite its simplicity, Zotero allows the user to associate a full set of metadata with images. Its combination of ease and power makes Zotero rewarding to teach, since it is both impressive and simple to demonstrate. Additionally, none of the other options let the users truly exploit their image metadata in useful ways. The main advantage to using Zotero is the potential to use that data to create maps, charts, and other digital humanities visualizations, or to generate citations, figure lists, and photo credits automatically.

While most other personal image managers are focused solely on images coming from digital cameras, Zotero is built for interaction with databases, and in some cases it can harvest images and their metadata from online collections. This functionality can add utility to local image databases, as well as satisfy image users' desire to coordinate institutional and personal collections. ${ }^{17}$ By quickly and easily bringing images into their own personal collections, users can tailor the organization and metadata to their own purposes. Using collections made of known items (personal libraries) produces very different information-seeking behavior than when using a collection made up of a yet-unknown body of images (image databases). ${ }^{\mathrm{I} 8}$ Zotero effectively creates a pipeline that efficiently moves images and their metadata from database to personal library, and then into a paper or presentation.

\section{ZOTERO FOR PERSONAL IMAGE MANAGEMENT}

ITEMS IN ZOTERO

There are two primary ways to bring images into a Zotero library. The most basic is to manually add a new artwork item, and then type in the metadata and attach an image file. Zotero can also add items and metadata from databases, including both Flickr and Wikimedia Commons; it will automatically download image files and attach the associated metadata. While this functionality is currently limited to these specific image databases, Zotero's open-source nature means that new databases can be made compatible with Zotero through the writing of a translator.

Zotero libraries are made up of item entries consisting of metadata with optional attached files. Users can attach multiple images to just a single record. This would make it easy to have a single entry with multiple images-for example, several views of the same object. It is also possible to have a separate record for each individual image, which is necessary if the user plans to cite them separately. Zotero is currently developing nested items, and in the future it should be possible to create overarching entries and metadata for specific photographs.

\section{ZOTERO'S METADATA}

Each different type of item in Zotero has its own metadata schema, though many fields are shared between item types. Zotero's artwork item type has fields for the most important image data (Figure 2). The ability to document images fully makes

I7. Pisciotta et al., "Penn State's Visual Image User Study," 35.

I8. Kerry Rodden and Kenneth R. Wood, "How Do People Manage Their Digital Photographs?," in Proceedings of the SIGCHI Conference on Human Factors in Computing Systems, CHI '03 (New York, NY, 2003), 409, doi:Io.II45/6426II.642682. 
Figure 2. Artwork item type metadata in Zotero.

\begin{tabular}{|c|c|l|l|}
\hline Info & Notes & Tags & Related \\
\hline Item Type: Artwork \\
Title: Apollo and Daphne \\
Artist: Bernini, Gian Lorenzo \\
Abstract: \\
Medium: Marble \\
Artwork Size: $243 \mathrm{~cm}$. \\
Date: $1622-1625$ \\
Language: \\
Short Title: \\
Archive: Borghese Gallery, Rome \\
Loc. in Archive: Room III \\
Library Catalog: \\
Call Number: \\
URL: \\
Accessed: \\
Rights: \\
Extra: \\
Date Added: Friday, March 08, 2013 4:38:22 PM \\
Modified: Sunday, March 10, 2013 2:56:43 PM \\
\end{tabular}

the program much more than a way to store and view images. It becomes a place to keep important information, generate citations, and make the data available for analysis. Zotero's metadata for images is strong compared to other image-management software programs, but it is less robust than full-featured digital asset management systems. It lacks the complexity of a schema like VRA Core or the ability to integrate vocabularies.

Zotero's metadata has always existed for practical purposes; the fields it includes are likely to be required in citations. Some users may find Zotero's set of metadata to be the right balance between too simple and overly complicated. Indeed, Penn State's study on metadata found that an elaborate schema was of little use, since the main challenge was finding sufficient information to populate the fields, not parsing overwhelming data. ${ }^{19}$

The artwork item type is used for all images, including paintings, threedimensional objects, architecture, or photographs. Users can enter an unlimited number of artists and can designate people as contributors rather than artists. Since Zotero was built by historians, the archive field is a product of that background. However, this potentially awkward field is a good fit for noting the holding museum, as well as the location of the work. Responding to this author's suggestion, in a future release Zotero may include a place field for artworks, a great example of how Zotero's

I9. Attig, Copeland, and Pelikan, "Context and Meaning," 257. 


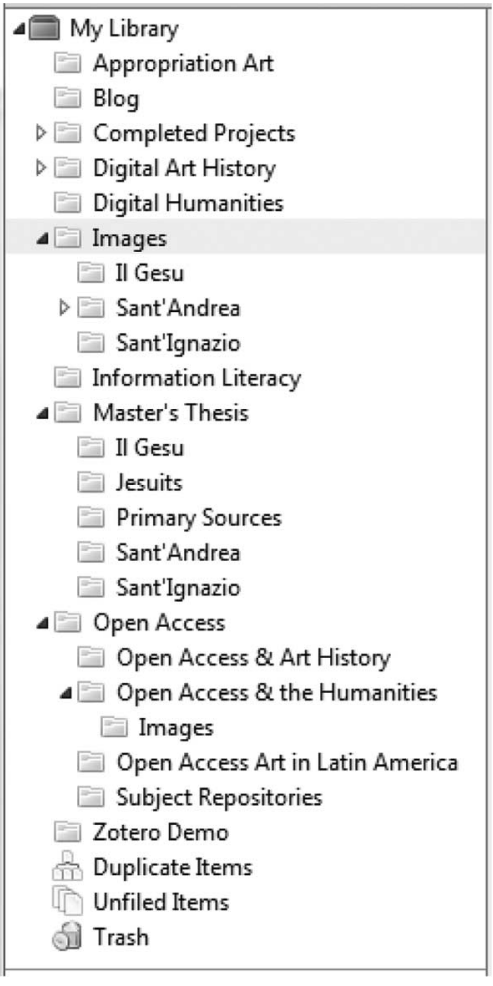

Figure 3. Zotero's library and collection folder system. Please see the online edition of Art Documentation for a color version of this image.

open-source development means it can react to the user community's needs. The library catalog field will be automatically populated from the database from which Zotero retrieved the image, such as Flickr. Another useful field is rights, which allows users to keep track of image rights and enables Zotero to generate photo credits. Finally, Zotero includes an extra field that can be used for any special metadata needs of the researcher.

ORGANIZING, SHARING, STORING

- Folders. Once image files are in Zotero, they can be organized in a number of ways. The main structure to bring order to Zotero entries is the folder system where items are sorted into a number of collections (Figure 3). They function more like iTunes playlists than a traditional folder system. One item can be in multiple places, and when edited, it changes everywhere. While this system has many benefits, it is not always intuitive. For example, when an item is dragged from one folder into another, it also stays in the original folder. This means that a single entry can be in multiple folders, allowing the user to set up folder systems that often repeat the same images, such as slide lists for similar classes. This eliminates the problematic choice inherent in many other systems when multiple categorizations apply that one must either 
Figure 4. Zotero-generated tag cloud. Please see the online edition of Art Documentation for a color version of this image.

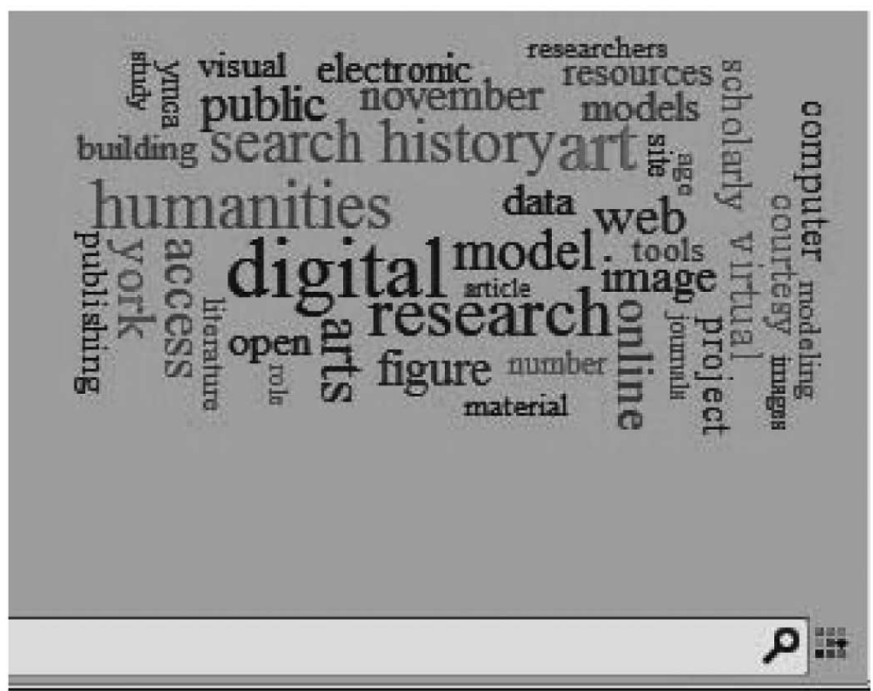

select only one category or create redundant copies in the corresponding folders. ${ }^{20}$

- Tags and relations. Zotero also allows users to employ tagging for organization. This lets the user have two concurrent organization systems when used with a folder system. Dual classification can alleviate the problem found in many image managers: no single organization policy is appropriate for all collections. Each time a new policy is required, the collection must be rearranged to satisfy the new criterion. ${ }^{2 \mathrm{I}}$ Instead, in Zotero items in separate folders can be brought together by tags (Figure 4). Often tags can be incorporated to keep track of workflow; images can be tagged, for example, with the phrase "needs permission." When the user is ready to seek permission for images, he or she can gather all the images together from across various image folders. Items can also be linked to one another using relations. Under the related metadata section one can simply select the other items in the library that are connected to the current item. This means one can link images to other views of the same object, related art works, or, because Zotero also is a repository for the user's research, to written sources about the item.

- Storing. When creating a Zotero library, the user is building a relational database. Images and files in Zotero are stored within its database file system; unfortunately, this means that there are no natural-language folders on the computer that the user can explore readily. Instead it is easiest to access the files through the Zotero program. Files that are added to Zotero can also be synchronized with Zotero.org's cloud storage. Signing up for an account is free, and it allows sharing of Zotero libraries. Three hundred megabytes of files can be stored on the Zotero server at no charge, and more storage is available for a fee.

20. Huang, Sumi, and Nishida, "Personal Image Repositories as Externalized Memory Spaces," I70. 2I. Ibid. 
Researchers will use the cloud storage mostly to access their files from any computer, but it can also serve as an extra backup of Zotero files.

- Sharing. Zotero lets users create group libraries that are shared among several people. Groups will let many users share or view the same set of images. This could make for an innovative alternative for sharing slides within a class setting. Users can also use groups to work collaboratively on an article or book, gathering images together alongside sources and citations. This could alleviate some of the silo phenomenon that happens when scholars rely solely on their personal image collections and duplicate work and effort. ${ }^{22}$

\section{ZOTERO AND IMAGE CITATION}

Zotero's main attraction to users has always been its ability to generate citations. Its plug-ins for Word and Open Office allow users to add footnotes, in-text citations, and bibliographies that can be formatted automatically in many different citation styles. ${ }^{23}$ Image citations pose a challenge because they take many forms and are often tricky or idiosyncratic. The major citation styles in Zotero, such as Chicago Manual of Style or MLA (Modern Language Association), can generate image citations, but they are usually not formatted correctly for image captions or figure lists. However, Zotero citations can be customized to create made-to-order formats.

Zotero formats citations based on styles created in the Citation Style Language (CSL), an open standard that is now used by many other citation managers including Mendeley and Papers. CSL styles can be created and edited using a graphical user interface (Figure 5) ${ }^{24}$ The editor has great potential, as users can specify and create unique in-text citation and bibliography styles. This means that librarians and visual resources specialists can create citation styles for images, while users could edit these to their precise specifications or make their own.

As a first step towards a robust system of image citation in Zotero, the author created a style called Chicago Manual of Style (figures \& illustrations) ${ }^{25}$ It is based on the Chicago Manual's instructions for citing figures ${ }^{26}$ and using this style with Zotero's Word plug-in allows users to create fully automatic figure citations. Using the toolbar seen in the upper left of Figure 6, users can search through the items in their Zotero library and insert them as in-line figure numbers. The advantage is that moving the image order or inserting new images will automatically change figure numbers throughout the document. When finished inserting in-line figure numbers, an accounting of all items used in the document can be generated as a list of illustrations, which will format the image metadata into Chicago style. While creating and refining

22. Kohl, "The Pedagogical and Research Value of Images and Locally Managed Image Collections," 53-55.

23. "Word Processor Plugin Installation [Zotero Documentation]," http://www.zotero.org/support/word_processor_plugin _installation.

24. "Citation Style Editor," http://editor.citationstyles.org/about/.

25. Alexander Watkins, "Figure and Illustration Citation Style for Zotero," http://alexwatkinsportfolio.wordpress.com/20I3/06/ I7/zotero-figure-list-figure-numbers-citation-style/.

26. The Chicago Manual of Style, I6th ed. (Chicago: The University of Chicago Press, 2010), sections 3.9, 3.22, 3.23, 3.27, and I4.I65. An additional source that informed this style was the College Art Association's "Guidelines for Correct Captioning of Images"; this document includes a good summary that is relevant to art history theses and papers: http://www.collegeart.org/ip/ guideforcorreccaps. 
Figure 5. Ordering citation elements in the graphical citation style editor. Please see the online edition of Art Documentation for a color version of this image.

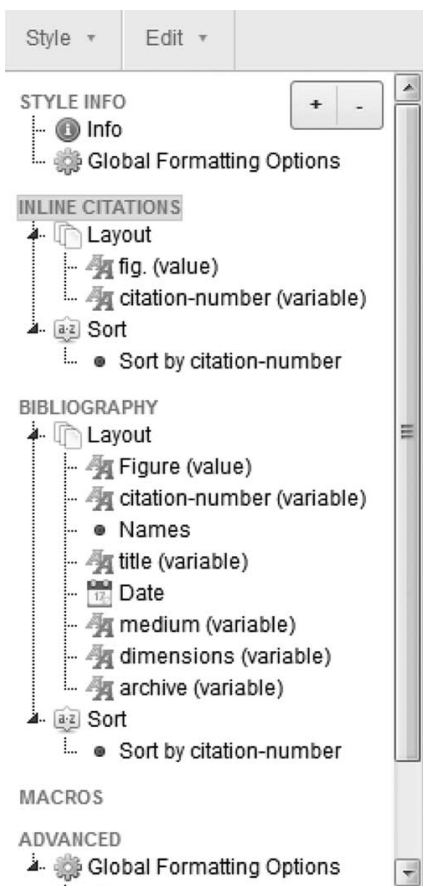

metadata is important in and of itself for organization and future reference, Zotero has a true advantage over other image-organization systems by being able to leverage that data for use.

To create and edit styles, users can take advantage of the CSL visual editor that works by building up citations from variables that map to Zotero metadata elements. ${ }^{27}$ Creating a new style for Zotero is straightforward; Figure 5 shows the user interface in the process of creating an image citation style. The necessary pieces of information for the citation are added as variables to the graphical interface in the order required by the style. For the figures \& illustrations style, the in-line citations were built with first the simple text "fig.," and then the variable "citation-number." Figure list citations are made of the text "Figure" along with the citation number with a period for a suffix. Each element of the citation is then added in order-in this example, author, title, date, medium, dimensions, and archive. For each variable the terminology in CSL is slightly different than the field names in Zotero; however, users can consult a crosswalk to select the correct term. ${ }^{28}$ For each variable, creators can choose styles like italics and delimiters such as commas, semicolons, periods, or parentheses, ultimately crafting a correctly formatted citation. For this style, following the Chicago Manual, commas separate the elements, and the title is in italics.

27. Zotero metadata elements are listed and mapped to CSL variables at "Zotero Item Types," http://aurimasv.github.com/ z2csl/typeMap.xml\#cslVar-dimensions.

28. Ibid. 


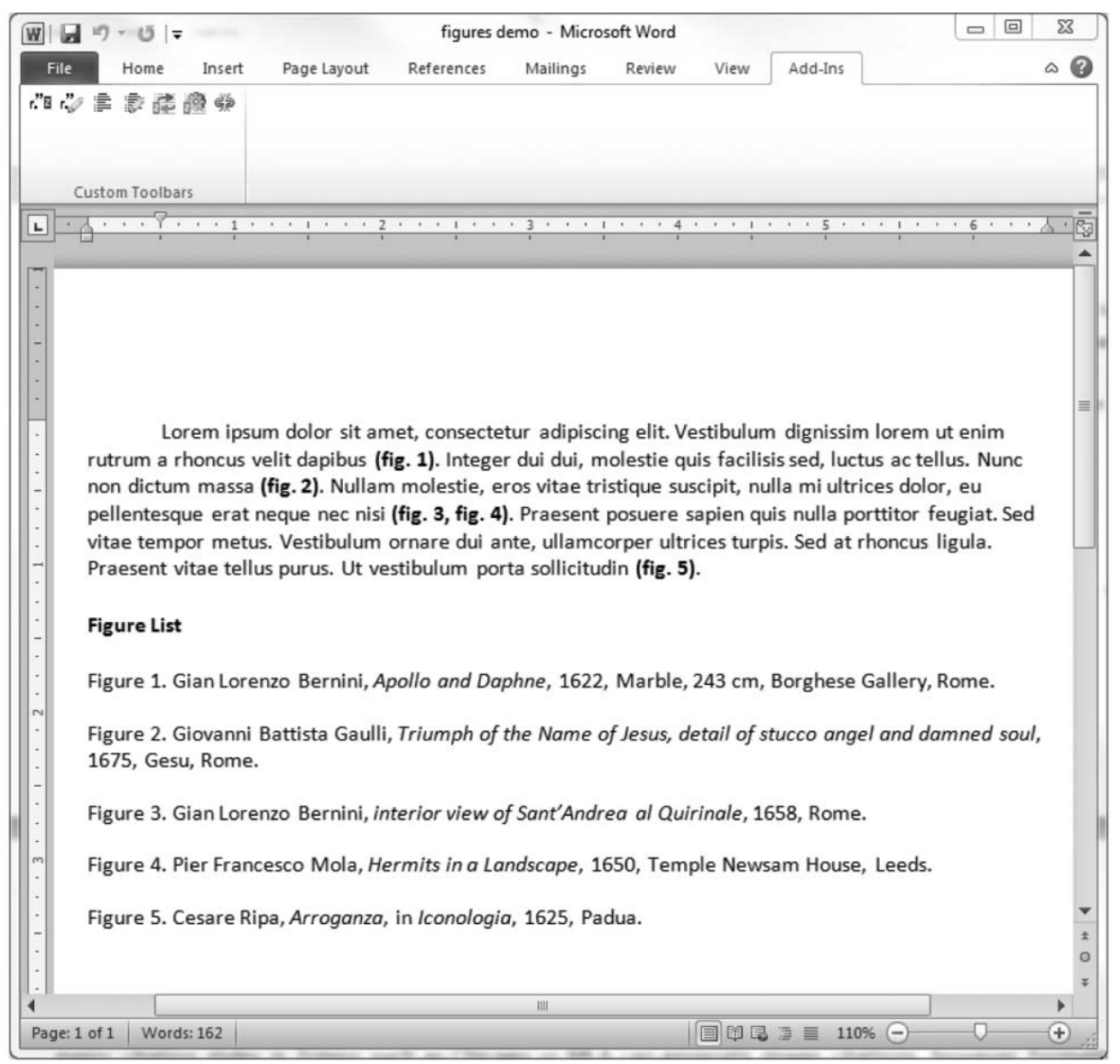

Figure 6. Figure citation style in action: figure list and in-text figure numbers generated automatically by Zotero using the toolbar in the upper left. Please see the online edition of Art Documentation for a color version of this image.

\section{ZOTERO AND IMAGE DATABASES}

Zotero is built around the ability to pull citations from library catalogs, article databases, or e-journals. Not only does this improve the utility of the sites themselves, it rewards high-quality metadata. It has the potential to shape how users conduct research, leading them to library catalogs and databases, locations that often have the best metadata. It uses coding called translators that tell Zotero how to pull information and files from individual databases, websites, or catalogs. Zotero already has translators for just about every article database. However, it has a paltry number of translators for images-as of this writing just Flickr and Wikimedia Commons. The translators are usually contributed by savvy users or by Zotero developers due to popular demand. It is hoped that the Zotero community can enter a virtuous cycle in which more image translators lead to more image users which lead to more translators. More Zotero compatible-image databases will mean that users will decreasingly have to enter their own metadata as they gather images. The 


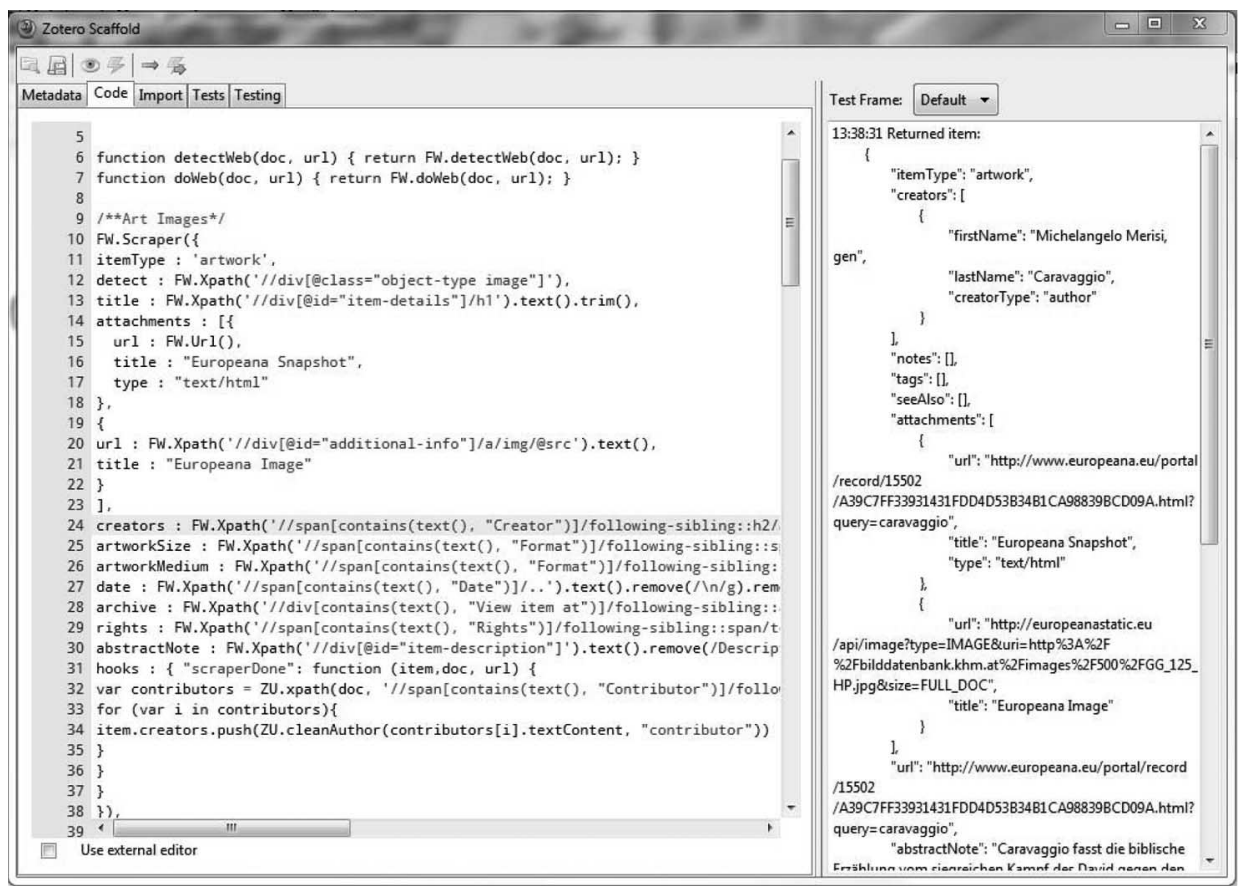

Figure 7. Building a translator in Scaffold. Please see the online edition of Art Documentation for a color version of this image.

power and ease of using Zotero translators are already clear when utilizing the program to gather article and book citations. Librarians and visual resources professionals are great candidates to get involved in creating translators for digital libraries and collections.

Creating translators takes a bit more programming expertise than creating a citation style. However, creating a "scraper" type translator, which simply pulls information out of the web page's HTML coding, can be done with just a basic knowledge of HTML and learning how to create Xpaths. The essential idea is that the metadata elements that are to be harvested are always located in the same place on each item's web page. If the creator can tell Zotero the path through the HTML to find where, for example, the title is contained, Zotero will be able to grab this information and import it. Adam Cymbal's guide for humanists on writing Zotero translators is the best place to start learning to create these useful tools. ${ }^{29}$ Translators are most easily built in a Firefox add-on called Scaffold (Figure 7), which automatically generates the enclosing coding and facilitates testing. ${ }^{30}$

The coding here requires equating Zotero metadata elements with Xpaths, which tell Zotero where on a web page to find the information to populate those elements.

29. Adam Crymble, "How to Write a Zotero Translator," http://niche-canada.org/member-projects/zotero-guide/chapterr.html. Crymble's guide has become out of date; Zotero maintains a wiki of updates here: "How to Write a Zotero Translator PlusPlus," http://www.zotero.org/support/dev/how_to_write_a_zotero_translator_plusplus.

30. "Scaffold—An IDE for Zotero Translators," http://www.zotero.org/support/dev/translators/scaffold. 
Using "detect," Xpaths also tell Zotero how to check if the page is Zotero-readable. They also instruct Zotero to download associated files via "attachments," allowing the coder to have Zotero automatically download image files. The Xpaths are defined by telling the program where to look on a web page by zeroing in on identifying characteristics of an HTML element. Although it can seem intimidating, once intrepid new coders have mastered the basic idea, they can capture just about any data on an HTML page and pass it through to Zotero. Additionally, the XCpath Bookmarklet will generate Xpaths easily from the browser. ${ }^{3 \mathrm{I}}$

The ability to create translators has great potential, because librarians and visual resources professionals can make their image databases and digital libraries Zoterocompatible. Libraries and visual resource centers put a great deal of time and effort into their images' metadata; if they also create a Zotero translator, this carefully crafted information can be mined and harnessed by users, adding value to the metadata. Translators help create a more porous transition from library resources into a personal image collection, a need identified in the Penn State study. ${ }^{2}$ In addition, as users create more organized and well-documented image libraries of their own, it becomes that much easier to feed those items back in the institution's image collection.

\section{CONCLUSION}

Digital images have revolutionized teaching, libraries, and visual resource centers. In an era of transformed services, art information professionals can add Zotero to their arsenal of support for their users' image-management needs. Zotero, while not a perfect resource for personal image libraries, strikes a balance between simplistic image browser and full-fledged asset management system. One of its greatest strengths is how it facilitates workflow from database to personal library to formatted citation.

More work will need to be done so that these transitions can be seamless, including more image translators and citation styles. Librarians and visual resource professionals are in a key position to create these tools. Many scholars and students are already familiar with how Zotero functions, and they may be more likely to adopt it as a solution because they are comfortable with the software and already have it installed. By facilitating personal image libraries, librarians and visual resources professionals can foster use of institutional databases and promote good digital record keeping, both vital to the longevity and continuity of unique and valuable personal image libraries.

3I. "XCPath Bookmarklet," http://dl.dropbox.com/u/84898I/it/xp/xp.html.

32. Pisciotta et al., "Penn State's Visual Image User Study," 35. 